\title{
BENEFICIAL EFFECTS OF NAFAZATROM ON ISCHEMIC REPERFUSED MYOCARDIUM
}

\author{
MICHAEL J. SHEA, JAMES J. MURTAGH, STANLEY R. JOLlY, GERALD D. ABRAMS, BERTRAM PITT and \\ BENEDICT R. LUCCHESI * \\ The University of Michigan Medical School, Departments of Pharmacology, Internal Medicine and Pathology, Ann Arbor, Michigan \\ 48109 , U.S.A.
}

Received 7 February 1984, accepted 13 March 1984

M.J. SHEA, J.J. MURTAGH, S.R. JOLLY, G.D. ABRAMS, B. PITT and B.R. LUCCHESI, Beneficial effects of nafazatrom on ischemic reperfused myocardium, European J. Pharmacol. 102 (1984) 63-70.

The effect of nafazatrom, a new antithrombotic agent, was studied in a canine model of regional myocardial ischemia. Nafazatrom was administered $1 \mathrm{mg} / \mathrm{kg}$ intravenously every $6 \mathrm{~h}$ for $48 \mathrm{~h}$. After $24 \mathrm{~h}$ of drug or placebo administration, animals underwent $90 \mathrm{~min}$ of occlusion of the proximal left circumflex coronary artery followed by gradual reperfusion over a period of $30 \mathrm{~min}$. Twenty-four hours later, the animals were sacrificed and infarct size was determined by histochemical staining with triphenyltetrazolium chloride. Nafazatrom-treated animals had a significant reduction in infarct size expressed as a percent of the anatomical area at risk for infarction: $21 \pm 5 \%$ in the treated group vs. $41 \pm 5 \%$ in the control group ( $\overline{\mathrm{X}} \pm$ S.E.M., $\mathrm{P}<0.05$ ). Histological examination confirmed the gross results of postmortem histochemical staining. Salvage of ischemically jeopardized tissue appeared to be unrelated to myocardial oxygen demand as there were no hemodynamic differences between groups. The beneficial effects of nafazatrom are presumably related to a limitation of autolytic processes on the heart during and after ischemia as a result of the drug's ability to inhibit lipoxygenase and to prevent the enzymatic degradation of prostacyclin.

Nafazatrom Myocardial ischemia Myocardial infarction

\section{Introduction}

Nafazatrom (BAYg6575) is a substituted pyrazole compound initially developed as an antithrombotic agent (Seuter et al., 1979). The initial studies with experimental models of thrombosis demonstrated successful limitation of thrombus formation despite an apparent lack of effect on hemodynamics, blood coagulation, fibrinolysis or in vitro platelet aggregation (Seuter et al., 1979). Vermylen and coworkers (1979), using serum from nafazatrom-treated volunteers, were able to demonstrate drug-stimulated release of prostacyclin from rat aorta slices. Inhibition of arterial throm-

\footnotetext{
* To whom all correspondence should be addressed: Department of Pharmacology, M6322 Medical Science Bldg. I, The University of Michigan Medical School, Ann Arbor, Michigan 48109 , U.S.A.
}

bosis and ex vivo platelet aggregation were shown subsequently in models of thrombosis involving the rabbit aorta (Buchanan et al., 1982) and the circumflex coronary artery of the conscious dog (Shea et al., 1984). Recently, nafazatrom has been characterized as having two apparently unrelated actions: (1) inhibition of the major catabolic enzyme of prostacyclin (Wong et al., 1982) and (2) inhibition of lipoxygenase, the enzyme which generates leukotrienes from arachidonic acid (Honn and Dunn, 1982).

We hypothesized that nafazatrom would have a beneficial effect in protecting myocardium at risk for infarction during an occlusion/reperfusion model of ischemic injury. We believed that the ischemic protection might be related to limitation of autolytic processes during and after ischemia owing to: (1) cytoprotection by prostacyclin (Vane, 1983) and/or, (2) limitation of myocardial damage 
by neutrophils (Romson et al., 1983) due to decreased leukotriene generation as a result of lipoxygenase inhibition.

\section{Materials and methods}

\subsection{Occlusion-reperfusion model of ischemic injury}

Thirty-three male mongrel dogs, $10-14 \mathrm{~kg}$, were anesthetized with i.v. sodium thiopental, $5-10 \mathrm{ml}$, to induce a $15 \mathrm{~min}$ period of anesthesia. Using aseptic surgical technique, catheters for drug infusion and arterial pressure monitoring were implanted in the left jugular vein and left carotid artery and exteriorized through the nape of the neck. The animals were allowed to recover and then received either nafazatrom, $1 \mathrm{mg} / \mathrm{kg}$, or diluent (saline plus $\mathrm{NaOH}$ ), according to a random numbers table. Nafazatrom was prepared by dissolving the drug in $20 \mathrm{ml}$ of saline adjusted to $\mathrm{pH}$ 9 with $0.1 \mathrm{~N} \mathrm{NaOH}$. Control diluent solutions were prepared in an identical fashion except for the absence of nafazatrom. The drug or control solution was administered intravenously every $6 \mathrm{~h}$ for a total of 8 doses $(48 \mathrm{~h})$. The dosage of nafazatrom and the dosing interval were chosen because of previous studies which indicated a beneficial effect of nafazatrom in a model of coronary thrombosis (Shea et al., 1984).

Twenty-four hours after vascular catheterization, the animals were anesthetized with pentobarbital sodium, $30 \mathrm{mg} / \mathrm{kg}$ intravenously, intubated and ventilated with room air using a mechanical respirator. Arterial pressure was monitored with a Statham P23Db pressure transducer and recorded along with lead II of the ECG on a Grass recorder. A left thoracotomy was performed and the heart was suspended in a pericardial cradle. The left circumflex coronary artery (LCCA) was isolated from surrounding tissues at a point just under the left atrial appendage, but proximal to any marginal branches. A variable mechanical occluder (Hosko et al., 1977) was placed around the LCCA and an electromagnetic flow probe (Carolina Instrument Co.) was positioned proximal to the occluder. By using the flow probe and adjusting the occluder, the point of critical stenosis was determined. This is defined as the point at which resting LCCA flow is unchanged; however, hyperemic response to a $10 \mathrm{~s}$ occlusion is eliminated. In this fashion, flow measurements were obtained in the preischemic period, at the point of establishing a critical stenosis and at 60 min after reperfusion in the absence of any stenosis. After the critical stenosis was determined, the occluder was left in position for $10 \mathrm{~min}$ after which it was adjusted to completely occlude flow for a period of $90 \mathrm{~min}$. The coronary blood flow then was gradually restored at $10 \mathrm{~min}$ intervals until the previously established point of critical stenosis was reached at $60 \mathrm{~min}$. At this point, the occluder and flow probe were removed and the chest incision was closed.

The present model of occlusion-reperfusion ischemic injury was used, as it was felt that the model represented the sequence of events that might occur in patients who are evolving a myocardial infarction and who are subjected to coronary artery thrombolysis. The period of occlusion was chosen so as to allow for irreversible myocardial injury which occurs between 20 and 60 min after occlusion (Reimer et al., 1977). We have added a reperfusion period which may resemble thrombolysis (spontaneous or iatrogenic) and/or release of vasospasm. The critical stenosis has been added for two reasons. By limiting reactive hyperemia after the release of the occlusion may mimic the response one might see in a human atherosclerotic vessel which is partially occluded with plaque in the basal state, becomes occluded and is then reperfused. Presumably, one sees reperfusion without reactive hyperemia. A second and more practical issue is that we wished to avoid the problem of hemorrhagic infarction and ventricular fibrillation which frequently are observed after uncontrolled reperfusion (Sheehan and Epstein, 1982).

After $24 \mathrm{~h}$ the electrocardiogram and blood pressure were monitored. The animals were reanesthetized, reintubated and placed on the respirator. The pericardial cradle was reestablished and LCCA flow was measured. Ventricular fibrillation was induced by $100 \mathrm{~Hz}$ DC current pulses applied to the ventricle after which the heart was removed. 


\subsection{Postmortem analysis of infarct size and histo- logic examination}

Myocardial infarct size and the anatomical area at risk for infarction were quantitated using a modification of a dual perfusion technique previously described (Romson et al., 1980) (fig. 1). A small bore cannula was inserted into the LCCA just distal to the site of the previous occlusion. A large bore cannula was inserted into the aorta above the aortic valve and the coronary ostia. The LCCA bed was perfused with a solution consisting of $1.5 \%$ triphenyltetrazolium chloride (TTC) in 20 $\mathrm{mM}$ potassium phosphate buffer $\left(\mathrm{pH} 7.4,37^{\circ} \mathrm{C}\right)$. The aorta was perfused in a retrograde manner with $0.5 \%$ Evans Blue. The simultaneous perfusions were made at a pressure of $100 \mathrm{mmHg}$ and maintained for 5 min while the heart was suspended in a water bath $\left(37^{\circ} \mathrm{C}\right)$. The heart was then sectioned from apex to base into $0.7 \mathrm{~cm}$ thick transverse slices. The areas of non-ischemic myocardium subserved by the left anterior de- scending coronary artery and right coronary artery are stained blue. The area of left ventricle at risk for infarction due to its dependence on the LCCA is identified by the lack of Evans blue staining, but instead, by the appearance of a brick-red color, due to the formazan dye resulting from the reduction of TTC by the dehydrogenases of viable myocardium. Within the anatomical area at risk of infarction is included the irreversibly injured areas which do not react with TTC and appear as a pale region which is distinguished easily for purposes of quantification. A diagram of a typical ventricular slice is shown in fig. 2. Ventricular sections were traced using clear plastic overlays for subsequent analysis of the extent of transmural infarction. The right ventricular, valvular and fatty tissues were dissected from the left ventricular sections and the remaining slices of left ventricle were dissected into areas of infarction, area at risk, and remaining left ventricle and weighed. Gravimetric analysis was employed for assessing the regions of interest as previous studies from this laboratory indi-

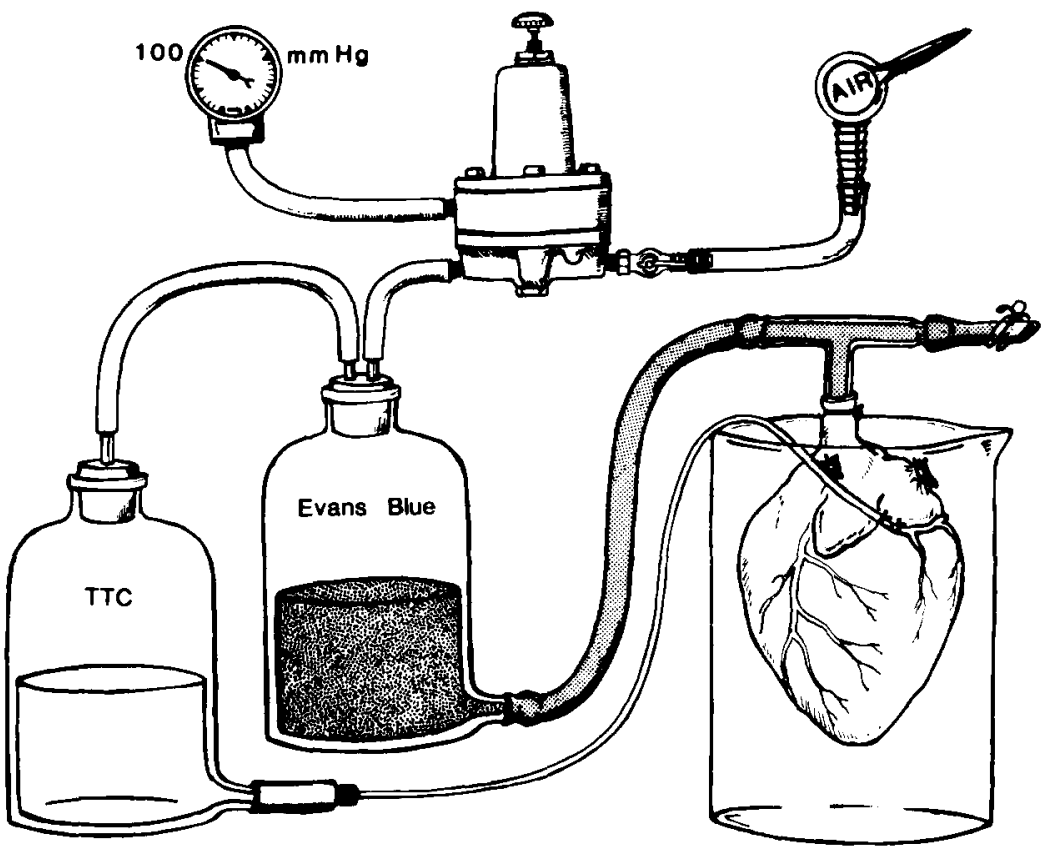

Fig. 1. A schematic diagram of the dual perfusion system used to delineate the left ventricle not at risk of infarction and the area at risk for infarction subserved by the left circumflex coronary artery (LCCA). The LCCA is perfused with triphenyltetrazolium chloride (TTC) while the aorta is perfused in a retrograde manner with Evans blue. Both the TTC and Evans blue reservoirs are maintained at a constant pressure of $100 \mathrm{mmHg}$. 


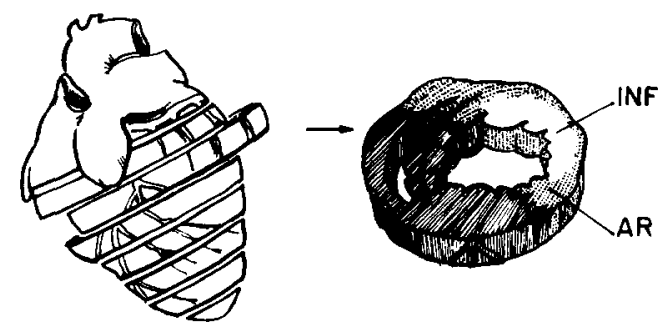

Fig. 2. A diagram of the differential staining of the heart. After perfusion with TTC and Evans blue, the heart is sectioned from apex to base. The lined areas include the area of left ventricle not at risk for infarction as well as the right ventricle (Evans blue positive). The stippled area indicates the area of the left ventricle supplied by the LCCA, the anatomic area at risk for infarction (TTC positive). The clear area within the stippled area at risk is the infarcted zone (fails to stain with TTC and appears pale)

cate that planimetric and gravimetric analysis of infarct/left ventricle $(\%)$ and area at risk/left ventricle (\%) closely agree (Bush et al., 1982; Jolly et al., 1982). Gravimetric analysis was performed by an individual unaware of the treatment regimen.

Tissue blocks were taken from the base of the heart including the posterior papillary muscle (an area dependent on the LCCA for blood supply and invariably infarcted in our studies), adjacent area at risk and an area of the left ventricle not at risk for infarction. Blocks were coded, fixed in $10 \%$ formalin, embedded in paraffin, cut into sections $7 \mu \mathrm{m}$ thick and stained with hematoxylin and eosin. An observer unaware of the treatment protocol analyzed the tissues qualitatively for the presence or absence of necrosis. He further attempted to assess the degree of leukocytic infiltration, hemorrhage and presence of microthrombi by using an arbitrary semiquantitative grading scheme of $0-4+$ where 0 would be the absence of microthrombi, homorrhage or leukocyte infiltration and $4+$ would be the densest accumulations of microthrombi, hemorrhage or leukocytes.

\subsection{Statistics}

All data are expressed as the means \pm S.E. unless otherwise stated. Student's $t$ test was used to determine the level of significance of differences between nafazatrom and control groups. Differences were considered significant when $P<0.05$.

\section{Results}

Of the initial 33 dogs randomized to drug or control treatment, three animals in each group were excluded because of heartworms, one animal from each treatment group was excluded because of technical error, three control animals were excluded because of no-reflow secondary to thrombotic occlusion of the LCCA at $24 \mathrm{~h}$ and two additional control animals did not complete the protocol; one due to ventricular fibrillation during occlusion of the LCCA and the other due to ventricular fibrillation during reperfusion. These latter two animals were the only ones which underwent multiple cardioversions for unresponsive ventricular arrhythmias. Two additional animals, one in each treatment group, were the only other animals requiring cardioversion, a single cardioversion in each case. The results from the remaining twenty animals form the basis for this report. The animals were comparable in terms of body weight, $12.9 \pm 0.4 \mathrm{~kg}$ and $13.2 \pm 0.6 \mathrm{~kg}$; and total left ventricular mass, $68.7 \pm 4.1 \mathrm{~g}$ and $73 \pm 6.4 \mathrm{~g}$, respectively, for treatment and control groups.

Nafazatrom- and drug-treated animals did not differ significantly with respect to heart rate, blood pressure or pressure-rate product during the baseline period or during occlusion, reperfusion, or at the $24 \mathrm{~h}$ time period (fig. 3). Mean coronary blood flows in the pretreatment and 60 min reperfusion time periods were similar for both groups: baseline flows were $20.2 \pm 1.6 \mathrm{ml} / \mathrm{min}$ and $23.0 \pm 2.4 \mathrm{ml} /$ min, respectively, for control and drug-treated groups. After $60 \mathrm{~min}$ of reperfusion the blood flows were $18.2 \pm 2.2 \mathrm{ml} / \mathrm{min}$ and $20.9 \pm 2.2 \mathrm{ml} /$ min, respectively, for control and drug-treated groups.

Nafazatrom significantly reduced infarct size as demonstrated in fig. 4 . There was a greater than $50 \%$ reduction of infarct size whether infarct mass was expressed as a percentage of the anatomical area at risk for infarction or infarction was expressed as a percentage of the total left ventricle. Of importance, the areas at risk for infarction were similar for both control and drug-treated groups (fig. 4).

Histological examination of the posterior papillary muscle sections confirmed results obtained 


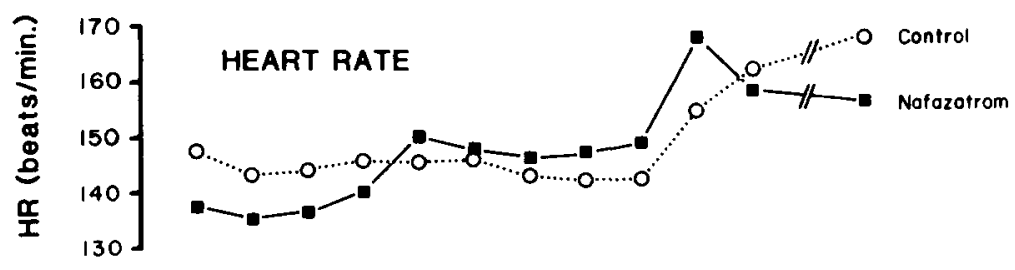

MEAN ARTERIAL PRESSURE
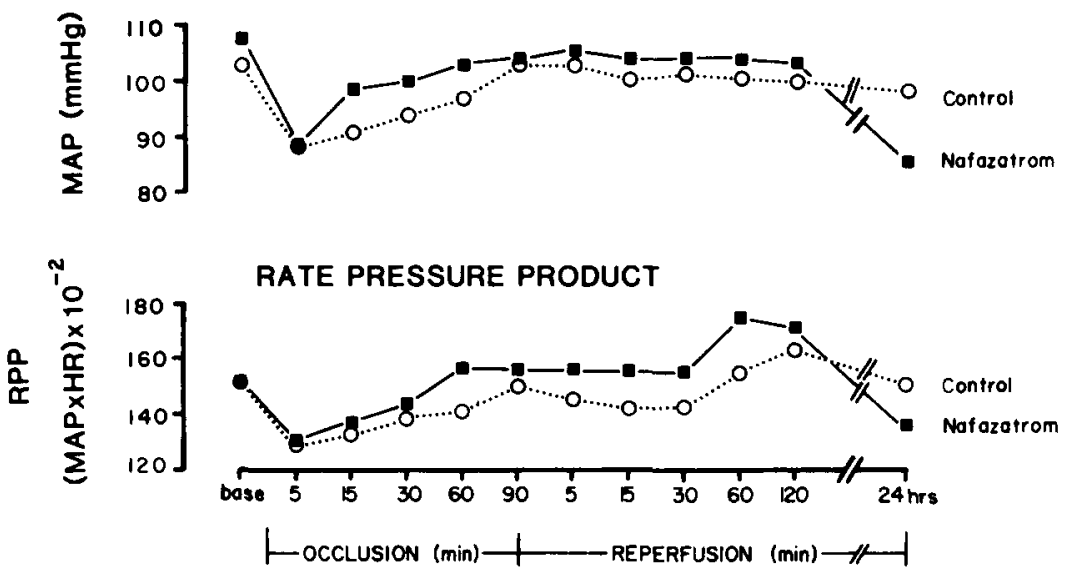

Fig. 3. Hemodynamic effects of nafazatrom during regional myocardial ischemia. The heart rate and mean arterial pressure were used to calculate the rate-pressure product. The time periods include the baseline, $90 \mathrm{~min}$ period of occlusion followed by reperfusion and subsequent measurements $24 \mathrm{~h}$ later. Not all animals were studied at $24 \mathrm{~h}$. Standard error bars are omitted for clarity.

from the gross examination after perfusion of the heart with TTC and Evans blue dye. Infarcted tissue; as determined by failure to react with TTC, demonstrated prominent contraction band necro-
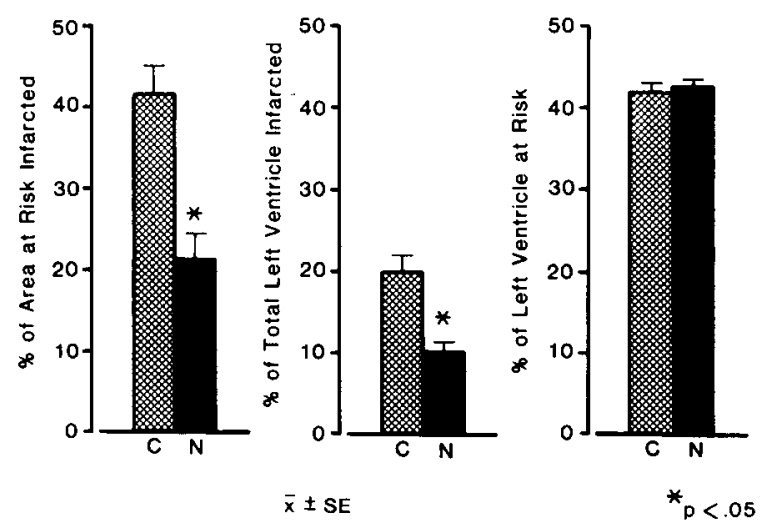

Fig. 4. Effect of nafazatrom on the extent of infarction expressed as a percent of the area at risk and as a percent of the left ventricle. The percent of the left ventricle occupied by the area at risk is demonstrated on the right. $\mathrm{C}=$ control $(\mathrm{n}=10)$; $\mathrm{N}=$ nafazatrom $(\mathrm{n}=10)$. sis, loss of nuclei and infiltration with inflammatory cells. There was no difference between nafazatrom-treated or control groups with respect to leukocytic infiltration or hemorrhage and no microthrombi were observed in either treatment groups as determined by light microscopy.

Gross visual examination of the infarcts indicated homogeneous areas of infarction with occasional small islands of adjacent infarcted tissue. From plastic overlay tracings, the transmural extent of infarction was assessed by projecting traces of ventricular sections and measuring the portions of the subendocardial and subepicardial surface circumference at risk of infarction and the portion of each surface that was irreversibly injured. The circumferential extent of subendocardial necrosis, expressed as percent of the circumference at risk, was $77 \pm 6$ percent in controls and $59 \pm 9$ percent in nafazatrom-treated hearts $(\bar{X} \pm$ S.E.M., $P>$ $0.05)$. The circumferential extent of epicardial necrosis was $19 \pm 6$ percent in controls and $3 \pm 2$ percent in nafazatrom-treated hearts $(\overline{\mathrm{X}} \pm$ S.E.M. 
$P<0.05$ ), suggesting that salvage of subepicardial myocardium was afforded by nafazatrom.

\section{Discussion}

This investigation indicates that nafazatrom significantly reduces the extent of myocardial injury at $24 \mathrm{~h}$ after a $90 \mathrm{~min}$ period of coronary artery occlusion followed by reperfusion. Nafazatrom has no significant effect on hemodynamics and thus does not alter myocardial oxygen demand in a favorable direction relative to control animals. LCCA coronary blood flow was similar both before and immediately after ischemia in both groups, however, the effect of nafazatrom on collateral blood flow is unknown. While nafazatrom is clearly beneficial in limiting the extent of myocardial injury, the mechanism of this beneficial effect is unclear, although several physiologic mechanisms are likely to be involved.

The blood platelet and platelet products, e.g., thromboxane, serotonin, etc., may have important roles in thrombosis and spasm of coronary blood vessels, responses which may be prevented with antiplatelet drugs (Haft et al., 1972; Kraikitpanitch et al., 1976). Our previous work with nafazatrom in a conscious canine model of coronary artery thrombosis demonstrated significant reductions in the incidence of epicardial coronary artery occlusive thrombosis (Shea et al., 1984). Fiedler (1983a) also has demonstrated antithrombotic activity with oral nafazatrom in a model of coronary thrombosis using the open-chest anesthetized dog. In our previous work ex vivo platelet aggregation studies indicated a significant antiplatelet effect which explained, in part, the reduction in platelet thrombi. Of interest in the present study was the presence of thrombotic occlusion of the LCCA in three control animals, but in none of the nafazatrom-treated animals. Insofar as nafazatrom has antiplatelet effects, the protection of ischemic myocardium may, in part, be a result of its antithrombotic action and the preservation of blood flow to the reperfused myocardium.

Recent work indicates that one of nafazatrom's primary actions is an inhibition of the major catabolic enzyme of prostacyclin, 15-OH pros- taglandin dehydrogenase, with a resultant increase in the local availability of prostacyclin (Wong et al., 1982). The use of prostacyclin for the protection of ischemic myocardial tissue has been demonstrated in previous models of myocardial ischemia in the cat (Ogletree et al., 1979) and the dog (Jugdutt et al., 1981). In the latter study, prostacyclin appeared to increase collateral blood flow to ischemic regions, however, some animals with low levels of collateral flow also had ischemic salvage arguing for other factors being important, e.g., cellular and metabolic effects. The relevance of these studies to our current investigation can not be directly extrapolated as most studies using prostacyclin for ischemic salvage have used pharmacologic doses involving a controlled hypotensive response. Nafazatrom has no apparent acute or chronic hemodynamic effects and the amount of prostacyclin generated appears to be hemodynamically insignificant (Vermylen et al., 1979).

An additional property of nafazatrom of potential benefit in myocardial ischemia is that of its ability to inhibit the lipoxygenase enzyme (Honn and Dunn, 1982). Lipoxygenase inhibition would decrease the generation of inflammatory leukotrienes with a resultant decrease in the migration of neutrophils to the reperfused and injured myocardium (Goetzl and Picket, 1980; Palmblad et al., 1981). The gross histologic appearance of hearts from control and drug-treated animals were similar. However, our histologic techniques are not sensitive enough to quantitatively assess neutrophil migration into previously ischemic areas. Recent studies from this laboratory have implicated inhibition of neutrophil migration to ischemically injured myocardium, assessed by the use of ${ }^{111}$ Indium-labeled neutrophils, in the beneficial action of ibuprofen on myocardial infarction (Romson et al., 1982). Furthermore, depletion of circulating neutrophils by administration of antibody against canine neutrophils was accompanied by a marked reduction in the ultimate extent of irreversibly injured myocardial tissue in the region undergoing reperfusion after a $90 \mathrm{~min}$ period of ischemia (Romson et al., 1983).

Another aspect of lipoxygenase inhibition with its attenuation of the inflammatory response likely involves a decrease in the formation of oxygen 
metabolites potentially injurious to the myocardium. This could occur either by a limitation of neutrophil-dependent inflammatory reactions which generate oxygen radicals (McCord, 1974; Fong et al., 1973; Johnston et al., 1973; Beauchamp and Fridovich, 1970) or by a direct free radical scavenging activity which recently has been reported for nafazatrom (Honn and Dunn, 1982).

An additional potential benefit of lipoxygenase inhibition would be a reduction of vasoconstrictor leukotrienes which have been shown to increase coronary vascular resistance in isolated heart preparations (Williams and Piper, 1980; Terashita et al., 1981).

Assessment of the patterns of infarction suggested that the salvage of myocardium was limited to the midepicardial and subepicardial layers in the nafazatrom-treated animals. These findings are compatible with previous investigations describing a wavefront phenomenon of cell death from subendocardium to subepicardium in which the border zone of potentially salvageable tissue is the subepicardial tissue (Reimer et al., 1977; Reimer and Jennings, 1979).

In this study, the effects of nafazatrom on ventricular arrhythmias were not assessed directly. In our previous study of coronary thrombosis, nafazatrom-treated animals had fewer ventricular arrhythmias than control animals after thrombosis (Shea et al., 1984). The suppression of ventricular arrhythmias also was confirmed by Fiedler (1983a) in a coronary thrombosis model. In a study of complete ligation of the rat anterior descending coronary artery, Fiedler showed that nafazatromtreated animals had fewer early and late ventricular arrhythmias as compared to controls (Fiedler, 1983b). Coker and Parratt (1983) used a $60 \mathrm{~min}$ occlusion followed by reperfusion in the anesthetized greyhound and were also able to show a significant inhibition of ventricular ectopic activity in nafazatrom-treated animals. Thus, nafazatrom may have antiarrhythmic activity, although the mechanism of action will require further investigation.

Our study indicates a significant benefit of nafazatrom in regional myocardial ischemia presumably due to a limitation of autolytic processes mediated by alterations of prostacyclin degrada- tion and leukotriene formation. Nafazatrom would be appealing to study in clinical ischemic situations as it has no apparent hemodynamic effects and thus could be given alone or in combination with other agents, in an effort to further modify the deleterious actions of neutrophils upon myocardial tissue undergoing reperfusion after a period of ischemia. Such a situation might exist in those instances where myocardial reperfusion is facilitated by coronary artery angioplasty or by thrombolytic therapy with streptokinase or tissue plasminogen activator.

\section{Acknowledgements}

This work was supported by the National Institutes of Health, Heart, Lung and Blood Institute, Grant No. HL-19782; HL-27817, by a grant from the Michigan Heart Association and by a grant from the Miles Research Institute. Michael J. Shea was a fellow of the Michigan Heart Association. Stanley R. Jolly was the recipient of a Pharmacology/Morphology Fellowship from the Pharmaceutical Manufacturer's Association Foundation.

\section{References}

Beauchamp, C. and I. Fridovich, 1970, A mechanism for the production of ethylene from methional. J. Biol. Chem. 245, 4641.

Buchanan, M.R., M. Blajchman and J. Hirsh, 1982, Inhibition of arterial thrombosis and platelet function by nafazatrom, Thromb. Res. 28, 157.

Bush, L.R., J.L. Romson, J.L. Ash and B.R. Lucchesi, 1982, Effects of diltiazem on extent of ultimate myocardial injury resulting from temporary coronary artery occlusion, $J$. Cardiovasc. Pharm. 4, 258.

Coker, S.J. and J.R. Parratt, 1983, Nafazatrom, prostacyclin and ZK366374 suppress reperfusion-induced ventricular fibrillation in anesthetized greyhounds, Br. J. Pharmacol. 79, 237.

Fiedler, V.B., 1983a, The effects of oral nafazatrom (BAYg6575) on canine coronary artery thrombosis and myocardial ischemia, Basic Res. Cardiol. 78, 266.

Fiedler, V.B., 1983b, Reduction of myocardial infarction and dysrhythmic activity by nafazatrom in the conscious rat, European J. Pharmacol. 88, 263.

Fong, K., P.B. McCay, J.L. Poyer, B.B. Keele and H. Misra, 1973, Evidence that peroxidation of lysosomal membranes is initiated by hyroxyl free radicals produced during flavin enzyme activity, J. Biol. Chem. 248, 7792.

Goetzl, E.J. and W.C. Pickett, 1980, The human PMN leuko- 
cyte chemotactic activity of complex hydroxyeicosatetraenoic acids (HETES), J. Immunol. 125, 1979.

Haft, J.L., K. Gershengorn, P.D. Kranz and R. Oestreicher, 1972, Protection against epinephrine-induced myocardial necrosis by drugs that inhibit platelet aggregation, Am. J. Cardiol. 30, 838.

Honn, K.V. and J. Dunn, 1982, Nafazatrom (BAYg6575) inhibition of tumor cell lipoxygenase activity and cellular proliferation, FEBS Lett. 139, 65 .

Hosko, M.J., G.J. Gross and D.C. Warltier, 1977, Technique for precise graded arterial stenosis and occlusion, Basic Res. Cardiol. 72, 651.

Johnston, R.B., B. Keele, L. Webb, D. Kessler and K.V. Rajagopalan, 1973, Inhibition of phagocytic bactericidal activity by superoxide dismutase: A possible role for superoxide anion in the killing of phagocytized bacteria, J. Clin. Invest. 52, 44a.

Jolly, S.R., G.D. Abrams, J.L. Romson, M.B. Bailie and B.R. Lucchesi, 1982, Effects of lodoxamide on ischemic reperfused myocardium, J. Cardiovasc. Pharmacol. 4, 441.

Jugdutt, B.I., G.M. Hutchins, B.H. Bulkley and L.C. Becker, 1981, Dissimilar effects of prostacyclin, prostaglandin $E_{1}$ and prostaglandin $E_{2}$ on myocardial infarct size after coronary occlusion in conscious dogs, Circ. Res. 49, 685.

Kraikitpanitch, S., C.C. Haygood, D.J. Baxter, A.A. Yunice and R.D. Lindeman, 1976, Effects of acetylsalicyclic acid, dipyridamole, and hydrocortisone on epinephrine induced myocardial injury in dogs, Am. Heart J. 92, 615.

McCord, J.M., 1974, Free radicals and inflammation: Protection of synovial fluid by superoxide dismutase, Science 185 , 529.

Ogletree, M.L., A.M. Lefer, J.B. Smith and K.C. Nicolau, 1979, Studies on protective effect of prostacyclin in acute myocardial ischemia, European J. Pharmacol. 56, 95.

Palmblad, J., C.L. Malmstein, A.M. Uden, O. Radmark, L. Engstedt and B. Samuelsson, 1981, Leukotriene $B_{4}$ is a potent and stereospecific stimulator of neutrophil chemotaxis and adherence, Blood 58, 658 .

Reimer, K.A. and R.B. Jennings, 1979, The wavefront phenomenon of myocardial ischemic cell death: transmural progression of necrosis within the framework of ischemic bed size (myocardium at risk) and collateral flow, Lab. Invest. 40, 633.
Reimer, K.A., J.E. Lowe, M.M. Rasmussen and R.B. Jennings, 1977, The wavefront phenomenon of ischemic cell death. 1 . Myocardial infarct size vs. duration of coronary occlusion in dogs, Circulation 56, 786.

Romson, J.L., L.R. Bush, D.W. Haack and B.R. Lucchesi, 1980, The beneficial effects of oral ibuprofen on coronary artery thrombosis and myocardial ischemia in the conscious dog, J. Pharmacol. Exp. Ther. 215, 271.

Romson, J.L., B.G. Hook, S.L. Kunkel, G.D. Abrams, M.A. Schork and B.R. Lucchesi, 1983, Reduction of the extent of ischemic myocardial injury by neutrophil depletion in the dog, Circulation 67, 1016.

Romson, J.L., B.G. Hook, V.H. Rigot, M.A. Schork, D.P. Swanson and B.R. Lucchesi, 1982, The effect of ibuprofen on accumulation of III-Indium labeled platelets and leukocytes in experimental myocardial infarction, Circulation 66, 1002.

Seuter, F., W.D. Busse, K. Meng, F. Hoffmeister, E. Moller and $\mathrm{H}$. Horstmann, 1979. The antithrombotic activity of BAYg6575, Arzneim. Forsch./Drug Res 29, 54.

Shea, M.J., E.M. Driscoll, J.L. Romson, B. Pitt and B.R. Lucchesi, 1984, The beneficial effects of nafazatrom on experimental coronary thrombosis, Am. Heart J. (in press).

Sheehan, F.H. and S.E. Epstein, 1982, Determinants of arrhythmia death due to coronary spasm: effect of pre-existing coronary artery stenosis on the incidence of reperfusion arrhythmia, Circulation 65, 259.

Terashita, Z.I., H. Fukui, M. Hirata, S. Terco, S. Olikawa, K. Nishikawa and S. Kikachi, 1981, Coronary vasoconstriction and $\mathrm{PGI}_{2}$ release by leukotrienes in isolated Guinea pig hearts, European J. Pharmacol. 73, 357.

Vane, J.R., 1983, Prostaglandins and the cardiovascular system, Br. Heart J. 49, 405.

Vermylen, J., D.A. Chamone and M. Verstraete, 1979, Stimulation of prostacyclin release from vessel wall by BAYg6575, an antithrombotic compound, Lancet 1, 518 .

Williams, T.J. and P.J. Piper, 1980, The action of chemically pure SRS-A on the microcirculation in vivo, Prostaglandins 19,779

Wong, P.Y.-K., P.H.W. Chao and J.C. McGiff, 1982, Nafazatrom (BAYg6575) an antithrombotic and antimetastatic agent, inhibits 15-hydroxy-prostaglandin dehydrogenase, J. Pharmacol. Exp. Ther. 223, 757. 